\title{
Conformally Symmetric Circle Packings: A Generalization of Doyle's Spirals
}

\author{
Alexander I. Bobenko and Tim Hoffmann
}

\section{CONTENTS}

1. Introduction

2. Geometry of Circle Flowers and Conformally Symmetric Circle Packings

3. Analytic Description of Conformally Symmetric Circle Packings

4. Doyle Spirals

5. Airy Functions as Continuous Limit

Acknowledgments

Electronic Availability

References
From the geometric study of the elementary cell of hexagonal circle packings - a flower of 7 circles - the class of conformally symmetric circle packings is defined. Up to Möbius transformations, this class is a three parameter family, that contains the famous Doyle spirals as a special case. The solutions are given explicitly. It is shown that these circle packings can be viewed as discretization s of the quotient of two Airy functions.

The online version of this paper contains Java applets that let you experiment with the circle packings directly. The applets are found at http:/www-sfb288.math.tu-berlin.de/Publications/ online/cscpOnline/Applets.html

\section{INTRODUCTION}

Circle packings (and more generally patterns) as discrete analogs of conformal mappings is a fast developing field of research on the border of analysis and geometry. Recent progress was initiated by Thurston's idea [1985] about the approximation of the Riemann mapping by circle packings. The corresponding convergence was proved by Rodin and Sullivan [1987]; many additional connections with analytic functions, such as the discrete maximum principle and Schwarz's lemma [Rodin 1987] and the discrete uniformization theorem [Beardon and Stephenson 1990], have emerged since then.

Circle packings constitute a natural topic for computer experimentation and visualization. Computer experiments demonstrate a surprisingly close analogy of the classical theory in the emerging "discrete analytic function theory" [Dubejko and Stephenson 1995]. Although computer experiments give convincing evidence for the existence of discrete analogs of many standard holomorphic functions, Doyle spirals (which are discrete analogs of the exponential function; see Section 4) are the only circle packings that have been described explicitly. 
Circle packings are usually described analytically in the Euclidean setting, that is, through their radii function. On the other hand, circles and tangencies are preserved by the fractional-linear transformations of the Riemann sphere (Möbius transformations). It is natural to study circle packings in this setting, i.e., modulo the group of the Möbius transformations. Z.-X. He and O. Schramm [1998] developed a conformal description of hexagonal circle packings, and used it to show that Thurston's convergence of hexagonal circle packings to the Riemann mapping is actually $C^{\infty}$. They describe circle packings in terms of the cross-ratios

$$
q(a, b, c, d):=\frac{(a-b)(c-d)}{(b-c)(d-a)}
$$

of their touching points.

Schramm [1997] introduced circle patterns with the combinatorics of the square grid (SG patterns). In many aspects the SG theory is analogous to the theory of the hexagonal circle packings. However, the SG theory is analytically simpler. The corresponding discrete equations describing the SG patterns, in the Euclidean as well as in the conformal setting, turn out to be integrable [Bobenko and Pinkall 1999]. Methods of the theory of integrable equations made it possible to find Schramm's circle patterns that are analogs of the holomorphic functions $z^{\alpha}$ and $\log z$ [Agafonov and Bobenko 2000]. Discrete $z^{2}$ and $\log z$ had been conjectured earlier [Schramm 1998] by Schramm and Kenyon.

One big question is which results on the Schramm's circle patterns carry over to the hexagonal setting, in particular whether some discrete standard functions can be described explicitly. This is closely related to the question of integrability of the basic discrete equations for hexagonal circle packings (the He-Schramm equation; see Section 3). In the present paper the first simple step in this direction is made. We study (surprisingly nontrivial) conformal geometry of hexagonal circle packings. In terms of this approach, a special class of conformally symmetric circle packings, which are generalizations of Doyle spirals, is introduced and all such packings are described explicitly.

Since this article deals with families of circle packings it seems natural to show not only arbitrarily chosen members in the figures, but to provide

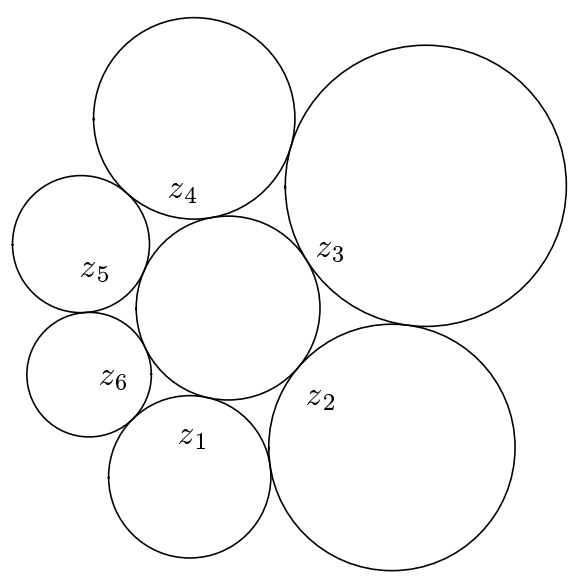

FIGURE 1. A circle flower (applet version available).

a possibility to present them all. Therefore there is an interactive version of this paper available online. It has some of the figures replaced by applets, which allow one to explore the families directly. See the section on Electronic Availability at the end for more information on this version.

\section{GEOMETRY OF CIRCLE FLOWERS AND CONFORMALLY SYMMETRIC CIRCLE PACKINGS}

This paper concerns patterns of circles in the plane called hexagonal circle packings. Their basic unit is the flower, consisting of a center circle tangent to and surrounded by petals. A hexagonal flower is illustrated in Figure 1; the six petals form a closed chain which wraps once in the positive direction (counterclockwise) about the center. Whereas neighboring petals touch, the circles of non-neighboring petals of a flower may intersect. We call a flower $\mathrm{im}$ mersed if none of its circles degenerates to a point. A hexagonal circle packing is a collection of oriented circles where each of its internal circles is the center of a hexagonal flower. Orientations of the circles should agree: at the touching points the orientations of the touching circles must be opposite. A hexagonal circle packing can be labeled by the triangular (hexagonal) lattice

$$
H L=n+m e^{i \pi / 3} \in \mathbb{C}, \quad \text { for } n, m \in \mathbb{Z} .
$$

or by one of its subsets. A circle packing is called immersed if all its flowers are immersed. Immersions of the whole $H L$ are called entire. Fractionallinear transformations of the complex plane (Möbius transformations) preserve circles, their orientation 
and their tangencies. In this paper we study circle packings modulo the group of Möbius transformations.

The center circle of a flower contains 6 points $z_{1}, \ldots, z_{6}$ (see Figure 1) where it touches the petals. We call them center touching points of a circle flower.

Proposition 2.1. Let $z_{1}, \ldots, z_{6}$ be points ordered counterclockwise on a circle $C$. The following three statements are equivalent:

(i) There exists a flower with the center $C$ and center touching points $z_{1}, \ldots, z_{6}$.

(ii) The multiratio $m$ of $z_{1}, \ldots, z_{6}$ is equal to -1 , that is,

$$
\begin{aligned}
m\left(z_{1}, z_{2}, z_{3}, z_{4}, z_{5}, z_{6}\right):= & \frac{\left(z_{1}-z_{2}\right)\left(z_{3}-z_{4}\right)\left(z_{5}-z_{6}\right)}{\left(z_{2}-z_{3}\right)\left(z_{4}-z_{5}\right)\left(z_{6}-z_{1}\right)} \\
& =-1
\end{aligned}
$$

(iii) There exists an involutive Möbius transformation $M$ (Möbius involution) such that

$$
M\left(z_{k}\right)=z_{k+3} \quad(k \bmod 6) .
$$

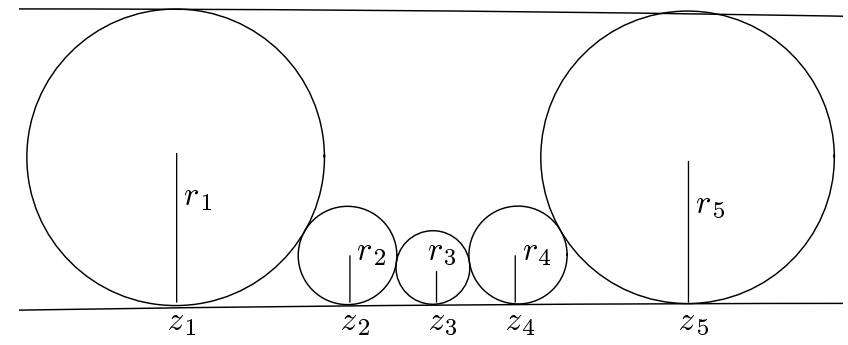

FIGURE 2. A flower with one central touching point at infinity.

Proof. Mapping the point $z_{6}$ to infinity by a Möbius transformation one obtains two parallel lines and five touching circles as in Figure 2. An elementary computation yields

$$
z_{k+1}-z_{k}=2 \sqrt{r_{k+1} r_{k}}, \quad \text { for } k=1, \ldots, 4,
$$

where $r_{k}$ are the radii of the corresponding circles. Together with $r_{1}=r_{5}$ and $\left(z_{5}-z_{6}\right) /\left(z_{6}-z_{1}\right)=-1$ this implies $(2-1)$.

On the other hand, given arbitrary $r_{1}>0$ and ordered $z_{1}, \ldots, z_{6}$ satisfying $(2-1)$, after normalizing $z_{6}=\infty$ formula $(2-2)$ provides us with the radii of the touching circles as in Figure 2. This proves the equivalence of (i) and (ii).
To show the equivalence of (ii) and (iii), define the Möbius transformation $M$ through $M\left(z_{1}\right)=z_{4}$, $M\left(z_{2}\right)=z_{5}, M\left(z_{3}\right)=z_{6}$. Consider $z_{*}=M\left(z_{4}\right)$. The invariance of the cross-ratios $q\left(z_{1}, z_{2}, z_{3}, z_{4}\right)=$ $q\left(z_{4}, z_{5}, z_{6}, z_{*}\right)$ implies the equivalence of $(2-1)$ and $z_{*}=z_{1}$. The same proof holds for $M\left(z_{5}\right)=z_{2}$ and $M\left(z_{6}\right)=z_{1}$.

To each center touching point $z_{k}$ of a flower, one can associate a circle $S_{k}$ passing through 4 touching points $z_{k-1}, z_{k+1}, w_{k}, w_{k-1}$ of the flower containing $z_{k}$ (see Figure 3). Here $w_{k}$ is the touching point of petals $\mathcal{P}_{k+1}$ and $\mathcal{P}_{k}$ (the petals are labeled by the corresponding touching points $z_{k}$ ). Indeed, mapping the point $z_{k}$ by a Möbius transformation to $\infty$, it is easy to see that the points $z_{k-1}, z_{k+1}, w_{k}, w_{k-1}$ are mapped to vertices of a rectangle, thus lie on a circle. We call these circles s-circles of a flower.

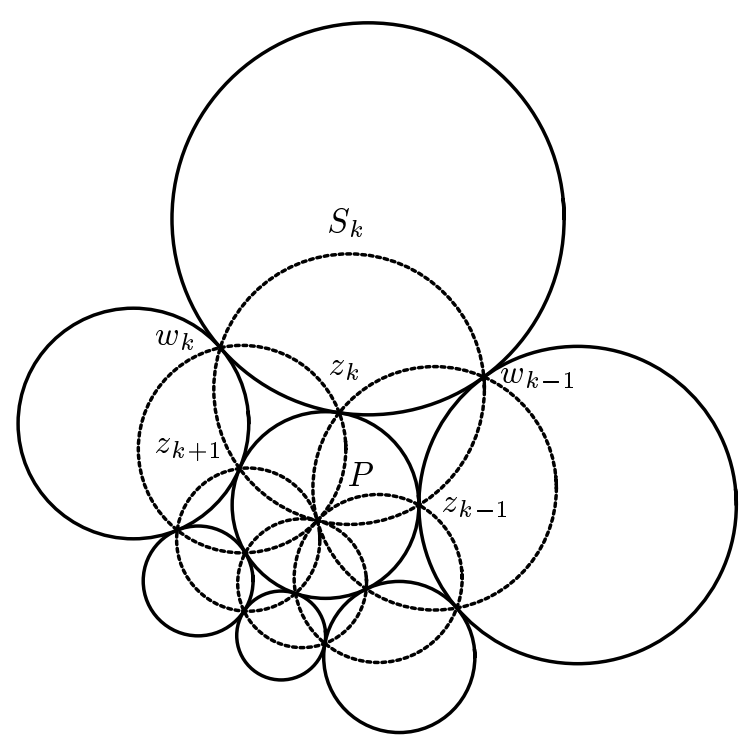

FIGURE 3. A conformally symmetric flower (applet version available).

Theorem 2.2. There exist a one-parameter family of flowers with the same center touching points. Moreover, there exists a unique flower $F$ in this family, which satisfies the following equivalent conditions:

(i) $F$ is invariant with respect to a Möbius involution $M$ with a fixed point $P$,

(ii) All s-circles of $F$ intersect in one point $P$.

We call the flower $F$ of the theorem conformally symmetric. 
One can view the whole family of flowers using an applet (see Electronic Availability at the end).

Proof. Keeping the points $z_{1}, \ldots, z_{5}$ in Figure 2 fixed and varying $r_{1}$ one obtains a one parameter family of flowers with the same touching central points. Let us now construct the flower $F$. The Möbius involution of Proposition 2.1 preserves the central circle $C$. Consider the circles $C_{k}$, for $k=1,2,3$, orthogonal to $C$ and passing through the pairs of points $\left\{z_{k}, z_{k+3}\right\}$. All these three circles intersect in 2 points $P$ and $P^{\prime}$, which are the fixed points of $M$ lying inside and outside $C$, respectively. By a Möbius transformation, map the point $P$ to infinity. The Möbius involution $M$ becomes $M(z)=-z$ and the circles $C_{1}, C_{2}, C_{3}$ become straight lines intersecting in the center of $C$. To construct the flower $F$, connect the $z_{k}$-points with even (respectively, with odd) labels by straight lines and consider their intersection points $w_{k}$ (see Figure 4 ). The circles $C_{k}$ passing through the triples $w_{k}, w_{k-1}, z_{k}$ touch at the points $w_{k}$. Let us prove this fact for $C_{1}$ and $C_{2}$. Indeed, the triangles $\Delta\left(w_{1}, w_{6}, z_{1}\right)$ and $\Delta\left(z_{3}, z_{5}, z_{1}\right)$ are similar, therefore the tangent lines to the circle $C_{1}$ at $w_{1}$ and to the circle $C$ at $z_{3}$ are parallel. The tangent lines to $C_{2}$ at $w_{1}$ and to $C$ at $z_{6}$ are also parallel. Since the points $z_{3}$ and $z_{6}$ are opposite on $C$, the circles $C_{1}$ and $C_{2}$ touch at $w_{1}$. The circles $C_{k}$

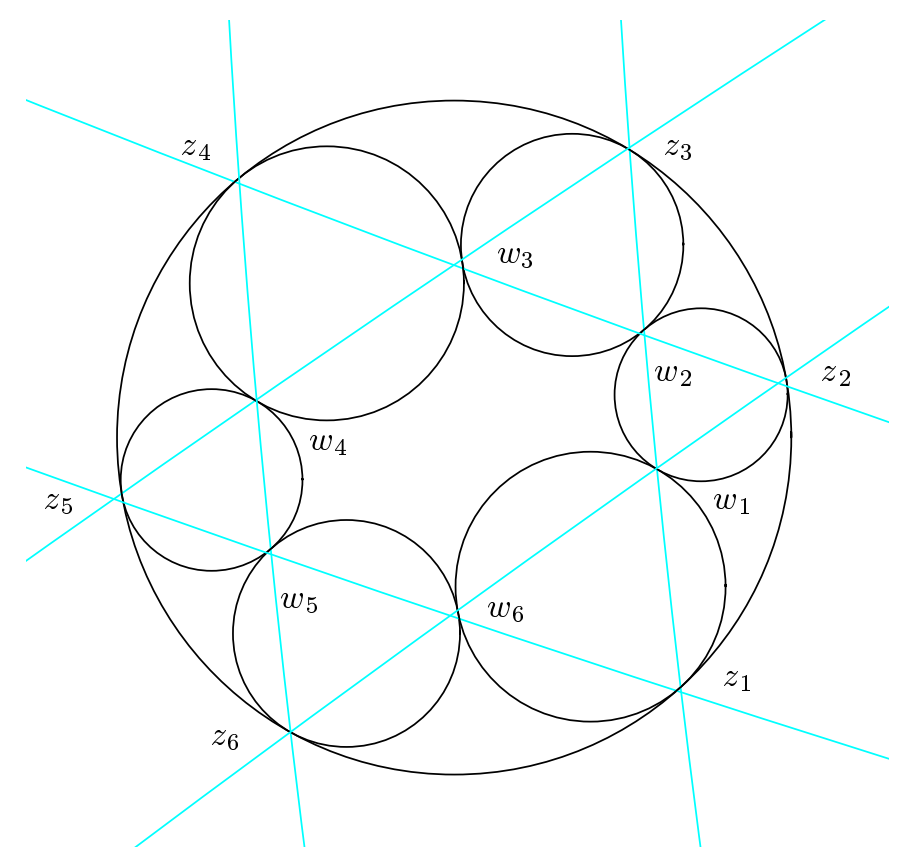

FIGURE 4. A normalized conformally symmetric flower. are the petals of the desired flower $F$, which is obviously $M$-symmetric. The s-circles of this flower are the straight lines $\left(z_{k}, z_{k+2}\right)$. The latter obviously intersect at infinity, thus all the s-circles of $F$ intersect in the fixed point $P$ of $M$.

The proof of (ii) $\Longrightarrow$ (i) is similar. After mapping the point $P$ to infinity the s-circles become straight lines and the flower is as in Figure 4. Since the circles in this figure touch, their tangent lines at the points $z_{k}, z_{k+3}$ and $w_{k+1}$ are parallel. This implies that $z_{k}$ and $z_{k+3}$ are opposite points on $C$, and the flower is symmetric with respect to the $\pi$-rotation of $C$.

Definition 2.3. A hexagonal circle packing is called conformally symmetric or an s-circle packing if it consists of conformally symmetric flowers; that is, if the s-circles of each of its flowers intersect in one point.

\section{ANALYTIC DESCRIPTION OF CONFORMALLY SYMMETRIC CIRCLE PACKINGS}

In this section we describe all conformally symmetric circle packings using the conformal description of circle packings proposed by He and Schramm [1998].

To each central touching point $z_{k}$ of a flower one associates the cross-ratio

$s_{k}:=q\left(z_{k}, z_{k-1}, w_{k-1}, w_{k}\right)=\frac{\left(z_{k}-z_{k-1}\right)\left(w_{k-1}-w_{k}\right)}{\left(z_{k-1}-w_{k-1}\right)\left(w_{k}-z_{k}\right)}$.

(Note that our normalization of $s_{k}$ differs from the one in [He and Schramm 1998].) Mapping $z_{k}$ to $\infty$, one observes that tree other points in $(3-1)$ are mapped to vertices of a rectangle, which implies that $s_{k}$ is purely imaginary. Moreover, the cross-ratios of an immersed oriented flower are positive imaginary, $-i s_{k}>0$. Also note that

$s_{k}=-q\left(z_{k+1}, z_{k-1}, w_{k-1}, z_{k}\right)=q\left(z_{k}, w_{k}, z_{k+1}, z_{k-1}\right)$,

and that $s_{k}^{2}=q\left(z_{k+1}, z_{k-1}, w_{k-1}, w_{k}\right)$ is the crossratio of the four touching points lying on the s-circle $S_{k}$.

Lemma 3.1. The cross-ratios $s_{k}$ of a flower satisfy the He-Schramm equation

$$
s_{k}+s_{k+2}+s_{k+4}+s_{k} s_{k+1} s_{k+2}=0
$$

for all $k \bmod 6$. 
Proof. Let $m_{k}$ be the Möbius transformation that takes $z_{k}, z_{k-1}, w_{k-1}$ to the points $\infty, 0,1$, respectively. By the definition of $s_{k}$ we have

$$
\begin{gathered}
s_{k}=q\left(z_{k}, z_{k-1}, w_{k-1}, w_{k}\right)=q\left(\infty, 0,1, m_{k}\left(w_{k}\right)\right), \\
-s_{k}=q\left(z_{k+1}, z_{k-1}, w_{k-1}, z_{k}\right)=q\left(m_{k}\left(z_{k+1}\right), 0,1, \infty\right) ;
\end{gathered}
$$

thus

$$
m_{k}\left(w_{k}\right)=1-s_{k}
$$

and

$$
m_{k}\left(z_{k+1}\right)=-s_{k}
$$

For $M_{k}:=m_{k+1} m_{1}^{-1}$ this yields $M_{k}\left(-s_{k}\right)=\infty$, $M_{k}(\infty)=0, M_{k}\left(1-s_{k}\right)=1$ and, finally,

$$
M_{k}=\left(\begin{array}{cc}
0 & 1 \\
1 & s_{k}
\end{array}\right)
$$

in the usual matrix notation for the Möbius transformations. The equality of the corresponding Möbius transformations implies

$$
M_{3} M_{2} M_{1}= \pm M_{4}^{-1} M_{5}^{-1} M_{6}^{-1},
$$

which is

$$
\begin{aligned}
& \left(\begin{array}{cc}
s_{2} & 1+s_{1} s_{2} \\
1+s_{2} s_{3} & s_{1}+s_{3}+s_{1} s_{2} s_{3}
\end{array}\right) \\
& = \pm\left(\begin{array}{cc}
-s_{4}-s_{6}-s_{4} s_{5} s_{6} & 1+s_{4} s_{5} \\
1+s_{5} s_{6} & -s_{5}
\end{array}\right) .
\end{aligned}
$$

Since the set of immersed flowers is connected and $s$ 's do not vanish the sign in this equation is the same for all flowers. Taking all the circles with the same radius one checks that the correct sign is plus, which implies the claim.

It is convenient to associate the touching points of a hexagonal circle pattern (as well as the cross-ratios $s_{k}$ ) to the edges of the honeycomb lattice. Equation $(3-3)$ is a partial difference equation on the honeycomb lattice. The cross-ratios on the edges of each hexagon satisfy (3-3). Moreover, it is easy to check that the He-Schramm equation is sufficient to guarantee the existence of the corresponding circle packing.

Proposition 3.2. Given a positive-imaginary function $s: E \rightarrow i \mathbb{R}_{+}$on the edges $E$ of the honeycomb lattice satisfying (3-3) on each honeycomb, there exists unique (up to Möbius transformation) immersed hexagonal circle packing with the cross-ratios given by the corresponding values of $s$.
Theorem 3.3. A circle flower is conformally symmetric if and only if its opposite cross-ratios $s_{k}$ are equal

$$
s_{k}=s_{k+3} \quad(k \bmod 6) .
$$

Proof. The property (3-4) for conformally symmetric flowers follows from (i) of Theorem 2.2. A simple computation with the flowers in Figure 4 shows that the map $\left(s_{1}, s_{2}\right)$ of immersed conformally symmetric flowers to $\left(i \mathbb{R}_{+}\right)^{2} \ni\left(s_{1}, s_{2}\right)$ is surjective. Since a flower is determined through the $s$ 's, the converse statement follows.

The general solution of $(3-3,3-4)$ on the whole $H L$ depends on three arbitrary constants and can be given explicitly. There is a Java applet that lets you explore this three parameter family of circle packings interactively (see section on Electronic Availability at the end.)

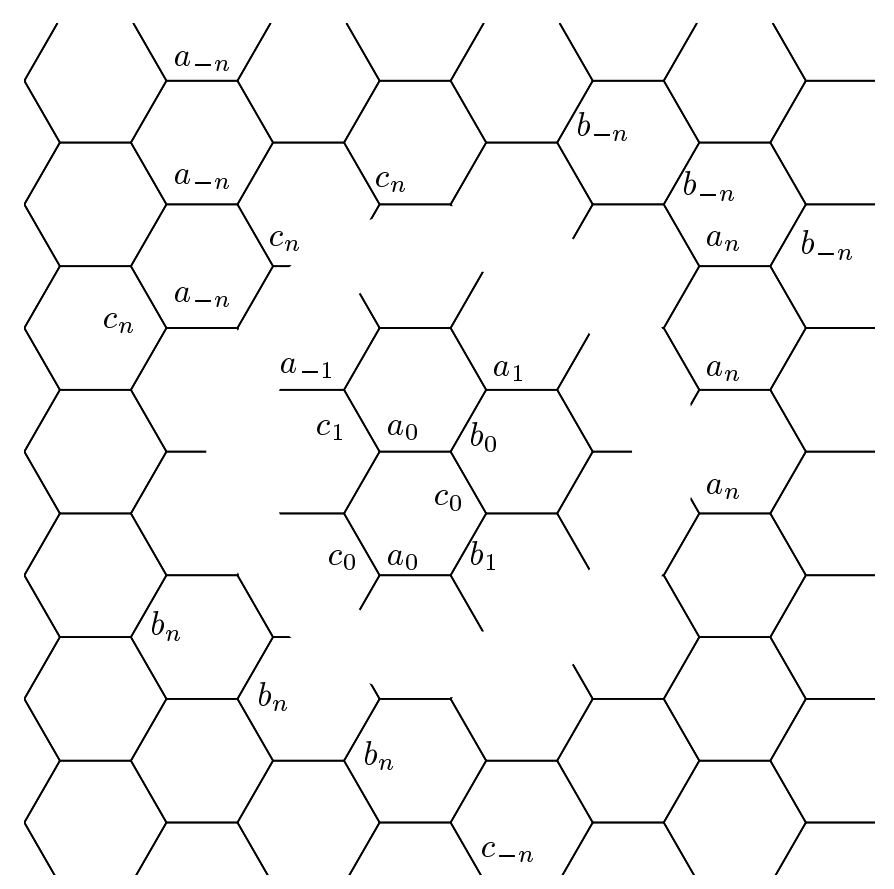

FIGURE 5. Cross-ratios of conformally symmetric circle patterns.

Theorem 3.4. The general solution of $(3-3,3-4)$ is given by

$$
\begin{aligned}
a_{n} & =i \tan (\Delta n+\alpha), \\
b_{n} & =i \tan (\Delta n+\beta), \\
c_{n} & =i \tan (\Delta n+\gamma),
\end{aligned}
$$

where $\Delta=-\alpha-\beta-\gamma$ and the cross-ratios $s_{k}$ on the edges of the hexagonal lattice are labeled by $a_{n}, b_{n}, c_{n}$ as shown in Figure 5 ( $n$ varies over the integers). 


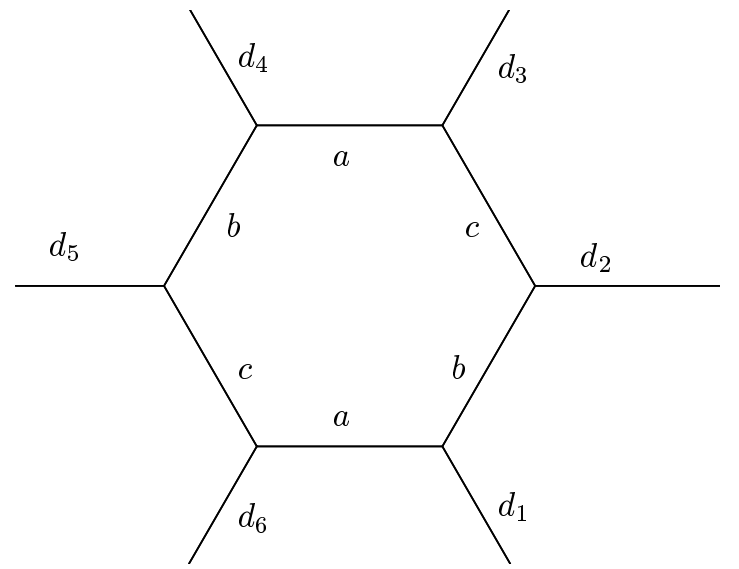

FIGURE 6. Continuation of conformally symmetric $s$ about a honeycomb.

Proof. We start with a simple proof of the consistency of the following continuation of a solution of (3-3)(3-4).

Given $s$ satisfying (3-3) and (3-4) on a honeycomb $H$, that is, $a, b, c$ in Figure 6 satisfying

$$
a+b+c+a b c=0,
$$

and a value of $s$ on one of the edges attached to the honeycomb (for example, $d_{1}$ in Figure 6), it can be uniquely extended to the full six honeycombs $H_{1}, \ldots, H_{6}$ neighboring $H$. Indeed, (3-3) and (3-4) yield

$$
b+d_{1}+d_{2}+b d_{1} d_{2}=0
$$

thus $d_{2}=M_{1}\left(d_{1}\right)$ is a Möbius transformation of $d_{1}$. Passing once around the honeycomb $H$ in this way one can check that (3-6) implies the monodromy Möbius transformation $M=M_{6} \ldots M_{1}$ is the identity, thus this continuation implies no constraints on $d_{1}$.

Proceeding this way, one reconstructs $s$ on the whole lattice $H L$ from its values on three adjacent edges $\left(a, b, d_{1}\right.$ above). Then (3-3) and (3-4) imply

$$
\begin{aligned}
a_{n}+b_{-n}+c_{1}+a_{n} b_{-n} c_{1} & =0, \\
a_{n+1}+b_{-n}+c_{0}+a_{n+1} b_{-n} c_{0} & =0
\end{aligned}
$$

and similar relations for other $a_{n}, b_{n}, c_{n}$. These identities become just the addition theorem for the tangent function, implying the formulas in $(3-5)$, which can be checked directly.

\section{DOYLE SPIRALS}

Denote by $R$ the radius of the center circle of a flower and by $R_{1}, \ldots, R_{6}$, the radii of its petals. Doyle spirals are characterized through the constraint (see [Beardon et al. 1994; Callahan and Rodin 1993] for a complete analysis of Doyle spirals)

$$
R_{k} R_{k+3}=R^{2}, \quad R_{k} R_{k+2} R_{k+4}=R^{3}
$$

on the radii of the circles (see Figure 7, where the central radius is normalized to be $R=1$ ). Doyle spirals have two degrees of freedom (for example the ratios $R_{1} / R$ and $R_{2} / R$, which are the same for the whole spiral) up to similarities. Again, you can experiment online with the two radii in a Java applet.

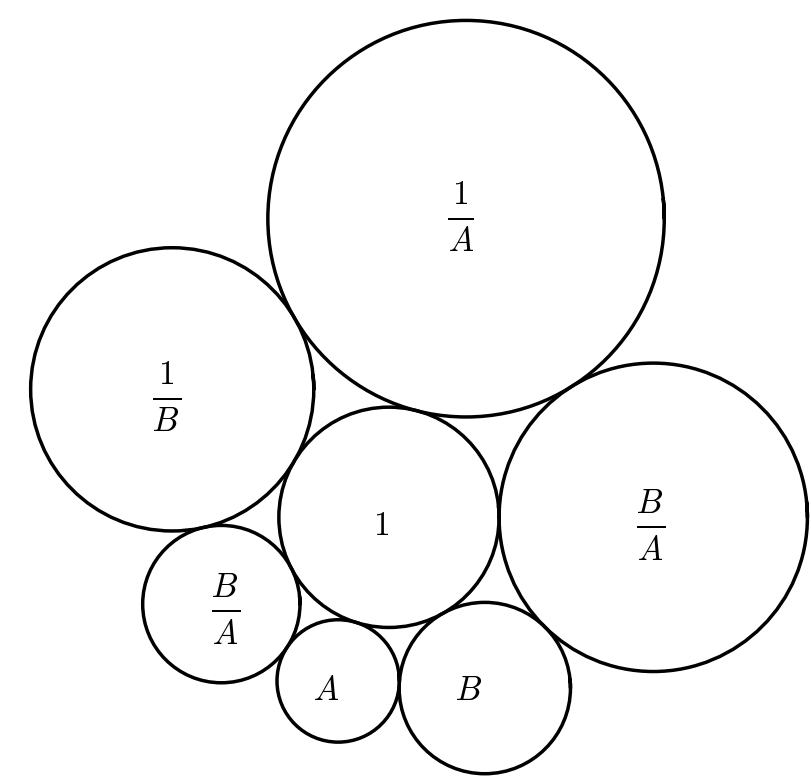

FIGURE 7. Radii of a Doyle spiral with the normalized central radius $R=1$ (applet version available).

Proposition 4.1. Doyle spirals are conformally symmetric.

Proof. The configurations of four touching circles with the radii $R, R_{k-1}, R_{k}, R_{k+1}$ and with the radii $R_{k+3}, R_{k+4}, R, R_{k+2}$ differ by scaling. This implies $s_{k}=s_{k+3}$ (use both (3-1) and the second representation of $s_{k}$ in (3-2)) and the claim follows by Theorem 3.3.

Theorem 4.2. Doyle spirals and their Möbius transformations can be characterized by the following two equivalent properties: 
(i) The circle packing is conformally symmetric, and the corresponding solution of (3-3) is "constant". It is of the form (3-5) with $\alpha, \beta, \gamma \in(0, \pi / 2)$ and $\alpha+\beta+\gamma=0(\bmod \pi)$ or, equivalently,

$$
\begin{array}{cl}
a_{n}=a_{0}, \quad & b_{n}=b_{0}, \quad c_{n}=c_{0}, \quad a_{0}, b_{0}, c_{0} \in i \mathbb{R}_{+}, \\
& a_{0}+b_{0}+c_{0}+a_{0} b_{0} c_{0}=0 .
\end{array}
$$

(ii) The whole circle packing is invariant with respect to the Möbius involution of each of its flowers.

Proof. All the flowers of a Doyle spiral differ by scaling, which implies (i). Consider the Doyle spiral as in Figure 7. Computing the cross-ratios through the radii, one shows that the map

$$
\begin{aligned}
\left\{(A, B) \in \mathbb{R}_{+}^{2}\right\} \rightarrow & \\
& \left\{(a, b, c) \in\left(i \mathbb{R}_{+}\right)^{3}: a+b+c+a b c=0\right\} .
\end{aligned}
$$

is surjective; thus (i) characterizes Doyle spirals and their Möbius transforms. The proof of the equivalence (i) $\Longleftrightarrow$ (ii) is elementary and is left to the reader.

It is an open problem whether Doyle spirals are the only entire circle packings. Formulas (3-5) imply that it is possible to have all cross-ratios being positive imaginary (necessary condition for entireness) only when $\Delta=0$.

Corollary 4.3. Doyle spirals are the only entire conformally symmetric circle packings.

\section{AIRY FUNCTIONS AS CONTINUOUS LIMIT}

Because of the property (4-1), Doyle spirals are interpreted as a discrete exponential function.

In the conformal setting this interpretation can also be easily observed. Indeed, let $P^{\varepsilon}$ be a family of circle packings approximating a holomorphic mapping in the limit $\varepsilon \rightarrow 0$. He and Schramm [1998] investigated the behavior of the cross-ratios $s_{k}$ in this limit:

$$
s_{k}=i \sqrt{3}\left(1+\varepsilon^{2} h_{k}^{\varepsilon}\right),
$$

where $h_{k}$ is called the discrete Schwarzian derivative (Schwarzian) of $P^{\varepsilon}$ at the corresponding edge of the hexagonal lattice. The discrete Schwarzians converge to the Schwarzian derivative

$$
S(f):=\left(\frac{f^{\prime \prime}}{f^{\prime}}\right)^{\prime}-\frac{1}{2}\left(\frac{f^{\prime \prime}}{f^{\prime}}\right)^{2}
$$

of the corresponding holomorphic mapping. More precisely, there exist continuous limits

$$
a=\lim _{\varepsilon \rightarrow 0} h_{1}^{\varepsilon}, \quad b=\lim _{\varepsilon \rightarrow 0} h_{2}^{\varepsilon}, \quad c=\lim _{\varepsilon \rightarrow 0} h_{3}^{\varepsilon}
$$

for the smooth functions $a, b, c$. (Note that we have $\lim _{\varepsilon \rightarrow 0} h_{k}^{\varepsilon}=\lim _{\varepsilon \rightarrow 0} h_{k+3}^{\varepsilon}$.) Because of (3-3) these functions satisfy

$$
a+b+c=0
$$

at each point. The Schwarzian equals

$$
S(f)=4\left(a+\omega^{2} b+\omega c\right), \quad \text { with } \omega=e^{2 \pi i / 3},
$$

and, using (5-2), this also yields

$$
\left.\begin{array}{rl}
6 a & =\operatorname{Re} S(f), \\
6 b & =\operatorname{Re}(\omega S(f)), \\
6 c & =\operatorname{Re}\left(\omega^{2} S(f)\right) .
\end{array}\right\}
$$

We see that, because of Theorem 4.2, Doyle spirals correspond to holomorphic functions with constant Schwarzian derivative $S(f)=$ const. The general solution of the last equation is the exponential function and its Möbius transformations.

It is natural to ask which holomorphic functions correspond to general conformally symmetric circle packings. In Figure 5 one observes that each of the cross-ratios $a_{n}, b_{n}, c_{n}$ is constant along one lattice direction. For the functions $a, b, c$ above, this implies

$$
\begin{aligned}
& a=a(\operatorname{Re} z), \\
& b=b(\operatorname{Re}(\omega z)), \\
& c=c\left(\operatorname{Re}\left(\omega^{2} z\right)\right),
\end{aligned}
$$

where $z$ is the complex coordinate. Comparing these equations with $(5-3)$ we see that the Schwarzian is a linear function of $z$ :

$$
S(f)=A z+B, \quad A \in \mathbb{R}, B \in \mathbb{C} .
$$

Equation (5-4) can be easily solved by standard methods. The general solution of $S(f)=u(z)$ with holomorphic $u(z)$ is given by

$$
f(z):=\psi_{1} / \psi_{2},
$$

where $\psi_{1}(z)$ and $\psi_{2}(z)$ are two independent solutions of the linear differential equation $\psi^{\prime \prime}=u(z) \psi$.

By a shift and scaling of the variable $z$, equation (5-4) with $A \neq 0$ can be brought to the form

$$
S(f)=z .
$$


As solutions of the corresponding linear equation

$$
\psi^{\prime \prime}=z \psi
$$

we have the Airy functions $\operatorname{Ai}(z)$ and $\operatorname{Bi}(z)$. On the real line the first of these is given by

$$
\operatorname{Ai}(x)=\frac{1}{\pi} \int_{0}^{\infty} \cos \left(x t+\frac{t^{3}}{3}\right) d t
$$

[Spanier and Oldham 1987], while the second is related to it by

$$
\operatorname{Bi}(z)=i q^{2} \operatorname{Ai}\left(\omega^{2} z\right)-i q \operatorname{Ai}(\omega z) .
$$

In the corresponding Möbius class of solutions of $(5-5)$ it is natural to choose

$$
f(z):=\frac{\operatorname{Bi}(z)-\sqrt{3} \operatorname{Ai}(z)}{\operatorname{Bi}(z)+\sqrt{3} \operatorname{Ai}(z)},
$$

which is the most symmetric one, $f(q z)=q f(z)$. The corresponding circle packing, symmetric with respect to the rotation $z \rightarrow q z$, is shown in Figure 8 .

Remark. One observes that the approximation in Figure 8 is excellent. On the other hand the results of Sections 3 and 4 imply that this approximation holds in finite domains only. For some large $n \in \mathbb{N}$, some cross-ratios become negative imaginary, which one can interpret as passing through infinity. Thus, the circle packing arrives at infinity for finite $n$. By refining the discretization - taking $\Delta \rightarrow 0$ in (3-5) — one can approximate the above-mentioned ratio of two Airy functions in an arbitrary finite domain.

As mentioned in the introduction, in connection with explicit examples, Schramm's SG-patterns are richer than the packings with hexagonal combinatorics. SG-patterns corresponding to ratios of two Airy functions were constructed in [Schramm 1997] (compare Figure 8.1.a of that paper with Figure 8). In contrast with our conformally symmetric circle packings, the Schramm circle patterns corresponding to them are entire, that is, they have regular behavior for all $(n, m) \in \mathbb{Z}^{2}$.

\section{ACKNOWLEDGMENTS}

The authors thank U. Hertrich-Jeromin, U. Pinkall, Yu. Suris and E. Tjaden for helpful discussions.

\section{ELECTRONIC AVAILABILITY}

Since the families of circle packings discussed in this paper have a finite (and even small) number of parameters, it seemed natural to look for a way to visualize whole families and experiment directly.

Java applets have been provided to illustrate the families of circle packing flowers, the whole class of conformally symmetric circle packings, and the special case of Doyle spirals. They also let the viewer wear "Möbius glasses", allowing the application of arbitrary Möbius transformation, which can help gain intuition. (Except for Doyle spirals, the families are only defined modulo an arbitrary Möbius transformation.) These applets can be found at http://www-sfb288.math.tu-berlin.de/Publications/ online/cscpOnline/Applets.html.

The page http://www-sfb288.math.tu-berlin.de/ Publications/online/cscpOnline/index.html includes these applets and the text of this paper, with instructions for the interactive figures. This online version renders a dvi file inside a Java applet. It requires a web browser that includes a Java virtual machine (any recent browser does). Note, however, that rendering the pages is slow on older machines; that's why we also provide the applets separately.

\section{REFERENCES}

[Agafonov and Bobenko 2000] S. I. Agafonov and A. I. Bobenko, "Discrete $z^{\gamma}$ and Painlevé equations", Internat. Math. Res. Notices 2000:4 (2000), 165-193.

[Beardon and Stephenson 1990] A. F. Beardon and K. Stephenson, "The uniformization theorem for circle packings", Indiana Univ. Math. J. 39:4 (1990), 13831425 .

[Beardon et al. 1994] A. F. Beardon, T. Dubejko, and K. Stephenson, "Spiral hexagonal circle packings in the plane", Geom. Dedicata 49:1 (1994), 39-70.

[Bobenko and Pinkall 1999] A. I. Bobenko and U. Pinkall, "Discretization of surfaces and integrable systems", pp. 3-58 in Discrete integrable geometry and physics, edited by A. I. Bobenko and R. Seiler, Oxford Lecture Ser. Math. Appl. 16, Oxford Univ. Press, Oxford, 1999.

[Callahan and Rodin 1993] K. Callahan and B. Rodin, "Circle packing immersions form regularly exhaustible surfaces", Complex Variables Theory Appl. 21:3-4 (1993), 171-177. 


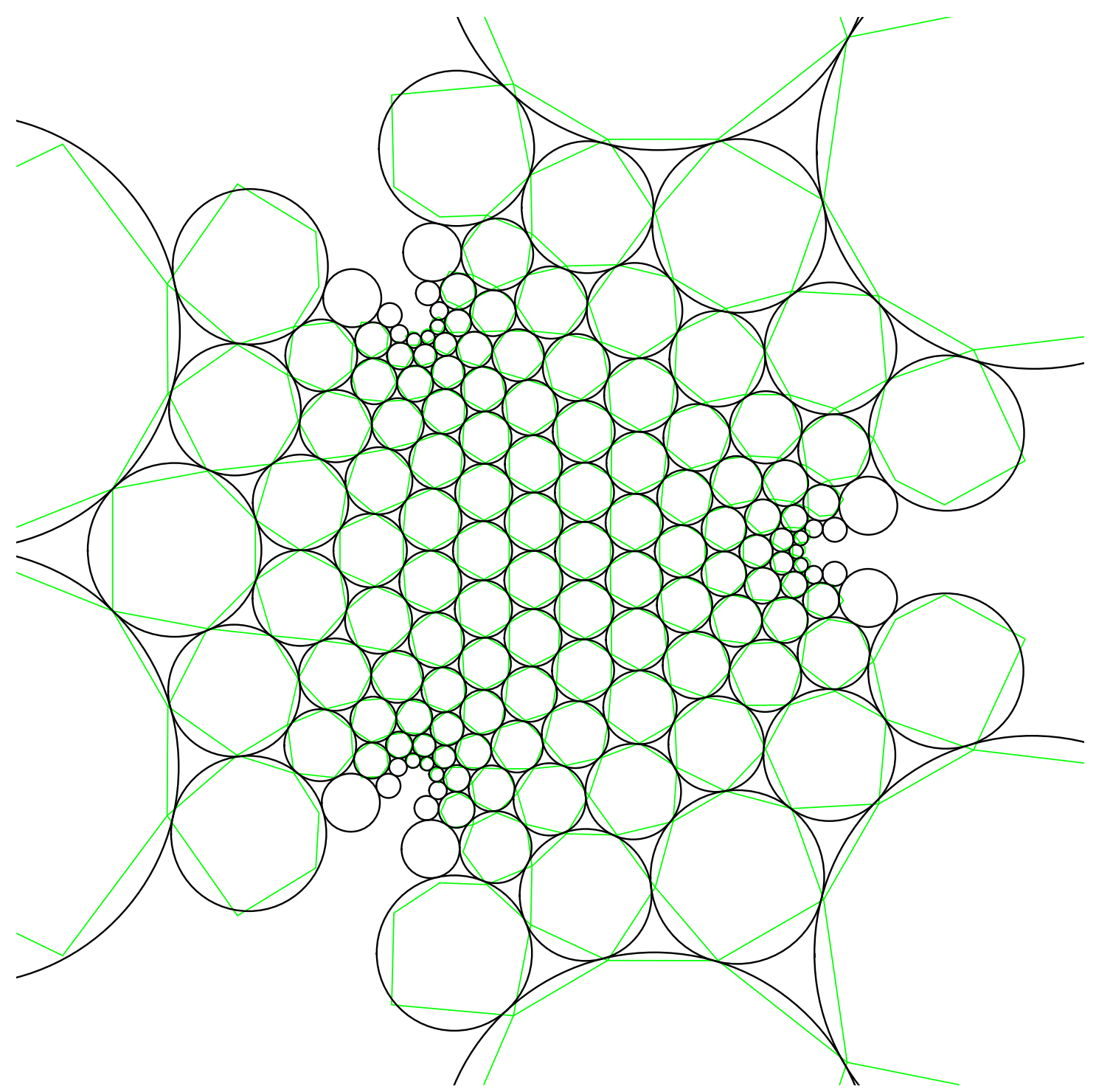

FIGURE 8. A conformally symmetric circle packing (with $\alpha=\beta=\gamma$ in (3-5)) and its smooth counterpart. The vertices of the hexagons are the images of the points of a standard hexagonal grid under the map $f$ from (5-6).

[Dubejko and Stephenson 1995] T. Dubejko and K. Stephenson, "Circle packing: experiments in discrete analytic function theory", Experiment. Math. 4:4 (1995), 307-348.

[He and Schramm 1998] Z.-X. He and O. Schramm, "The $C^{\infty}$-convergence of hexagonal disk packings to the Riemann map", Acta Math. 180:2 (1998), 219245.

[Rodin 1987] B. Rodin, "Schwarz's lemma for circle packings", Invent. Math. 89:2 (1987), 271-289.

[Rodin and Sullivan 1987] B. Rodin and D. Sullivan, "The convergence of circle packings to the Riemann mapping", J. Differential Geom. 26:2 (1987), 349-360.
[Schramm 1997] O. Schramm, "Circle patterns with the combinatorics of the square grid", Duke Math. J. 86:2 (1997), 347-389.

[Schramm 1998] O. Schramm, "Circle packings and conformal geometry, a survey of selected topics", 1998. See http://www.math.weizmann.ac.il/ schramm/talks.

[Spanier and Oldham 1987] J. Spanier and K. B. Oldham, An atlas of functions, Hemisphere Pub. Corp., Washington, 1987.

[Thurston 1985] W. P. Thurston, "The finite Riemann mapping theorem", 1985. Invited address, International Symposium in Celebration of the Proof of the Bieberbach Conjecture, Purdue University. 
Alexander I. Bobenko, Fachbereich Mathematik, Technische Universität Berlin, Str. 17 Juni 136, 10623 Berlin, Germany (bobenko@math.tu-berlin.de)

Tim Hoffmann, Fachbereich Mathematik, Technische Universität Berlin, Str. 17 Juni 136, 10623 Berlin, Germany (timh@sfb288.math.tu-berlin.de)

Received July 11, 2000; accepted in revised form September 9, 2000 